\title{
RECRUTEMENT DES CIVELLES (ANGUILLA ANGUILLA) SUR LA COTE MEDITERRANEENNE FRANÇAISE : ANALYSE COMPAREE DES CARACTERISTIQUES BIOMETRIQUES ET PIGMENTAIRES DES SAISONS 1974-75 ET 2000-01.
}

\author{
F. LeFEBVRE (1), E. SERGENT (1), A. ACOU (1), R. LECOMTE-FINIGER (2),
} A. J. CRIVELLI (1)

(1) Station Biologique de la Tour du Valat, Le Sambuc, 13200 Arles, France.

E-mail : a.crivelli@tourduvalat.org

(2) Laboratoire d'Ichtyoécologie Tropicale et Méditerranéenne, EPHE-CNRS, ESA 8046, Université de Perpignan, 66860 Perpignan Cedex, France.

E-mail : lecomte@univ-perp.fr

\section{RESUME}

Une campagne d'échantillonnage des civelles Anguilla anguilla a été menée de novembre 2000 à mai 2001 au grau de la Fourcade (Saintes-Maries-de-la-Mer, côte méditerranéenne française). Des civelles ont été capturées sur toute la période d'échantillonnage, mais les maxima d'abondance se situent en janvier-février et, dans une moindre mesure, en avril. Afin d'optimiser le recrutement dans les lagunes littorales intérieures, la mesure de gestion proposée est donc de favoriser l'ouverture des martelières au milieu de l'hiver.

Le suivi des proportions mensuelles des différents stades pigmentaires (de V A à VI $\mathrm{A}_{4}$ ) permet de conclure à un vieillissement généralisé des civelles en recrutement de novembre à mars, puis à l'arrivée d'un deuxième flux de civelles jeunes en avril. Parallèlement, il est observé une diminution très nette des masses et des longueurs moyennes mensuelles, et ce, même en ne considérant qu'un stade pigmentaire donné (en l'occurrence V B). Ces résultats sur l'évolution des caractéristiques biométriques et pigmentaires sont en accord avec les deux séries de travaux déjà menés sur la façade méditerranéenne française (années 30 et 70), ainsi qu'avec les données publiées en différents points de la façade atlantique.

L'analyse comparée de ces données avec celles issues de la dernière étude en date sur la côte méditerranéenne française (Bages-Sigean, Languedoc-Roussillon, campagne 1974-75 ; LECOMTE-FINIGER, 1976) montre une composition pigmentaire mensuelle totalement différente, et révèle une diminution significative de la longueur des civelles, de l'ordre de $5 \%$ en 25 ans.

Mots-clés : civelle, Anguilla anguilla, recrutement, biométrie, pigmentation. 


\title{
RECRUITMENT OF GLASS EELS (ANGUILLA ANGUILLA) \\ ON THE FRENCH MEDITERRANEAN COAST: \\ A COMPARATIVE ANALYSIS OF BIOMETRIC AND PIGMENTATION \\ CHARACTERISTICS DURING THE 1974-75 AND 2000-01 SAMPLING SEASONS.
}

\begin{abstract}
A sampling of glass eels, Anguilla anguilla, took place at the Fourcade sluice (Saintes-Maries-de-la-Mer, French Mediterranean coast) between November 2000 and May 2001. Glass eels were captured throughout the sampling period but abundance maxima occur in January-February and, to a lesser degree, in April. In order to optimise recruitment in the interior coastal lagoons, the suggested management measure would therefore be to open the sluice gates in mid-winter.
\end{abstract}

The monitoring of monthly proportions of different pigmentation stages (from $\mathrm{VA}$ to $\mathrm{VI} \mathrm{A}_{4}$ ) allows to conclude that there is a general aging of recruited glass eels from November to March followed by a second influx of young glass eels in April. At the same time, a clear reduction in mean monthly masses and lengths is observed even when considering a single pigmentation stage ( $\mathrm{V} B$ ). These results on the evolution of biometric and pigmentation characteristics support the conclusions of studies already carried out on the French Mediterranean coast (1930's and 1970's) as well as published data from different sites on the Atlantic coast.

A comparative analysis of these data with those resulting from the most recent study on the French Mediterranean coast (Bages-Sigean, Languedoc-Roussillon, 197475, LECOMTE-FINIGER, 1976) shows a completely different monthly pigmentation composition and reveals a significative decline in the length of glass eels in the range of $5 \%$ over 25 years.

Key-words: glass eels, Anguilla anguilla, recruitment, biometry, pigmentation.

\section{INTRODUCTION}

Sur la côte méditerranéenne française, l'anguille est traditionnellement la principale espèce piscicole exploitée (LECOMTE-FINIGER et BRUSLÉ, 1984 ; CRIVELLI, 1998). Cette ressource représente à elle seule environ $70 \%$ du revenu annuel des pêcheurs professionnels des lagunes littorales (QUIGNARD et al., 1983 ; DUSSERRE et LOSTE, 1997).

Depuis le début des années 80 , tant au niveau local qu'au niveau européen, il est constaté une sévère diminution des captures d'anguilles jaunes et argentées (MORIARTY et DEKKER, 1997 ; CRIVELLI, 1998). Bien qu'il soit difficile d'établir un lien direct entre le stock et le recrutement, les effectifs de civelles arrivant sur les côtes européennes amorcent eux aussi un sévère déclin (DESAUNAY et GUERAULT, 1997). En fait, concernant la civelle, deux tendances apparaissent à l'échelle européenne : une diminution d'abondance (partout où des données sont disponibles) et une diminution globale de la taille (longueur et masse). Les principaux travaux qui font état de ce constat ont été menés sur la façade atlantique (DESAUNAY et GUERAULT, 1997, et références incluses). Sur le pourtour méditerranéen, des informations sur la civelle, bien que moins abondantes, sont également disponibles en différentes localités (Maroc : YAHYAOUI, 1988 ; YAHYAOUI, 1991 ; Tunisie : HELDT et HELDT, 1929 ; Egypte : EZZAT et EL SERAFY, 1977 ; Italie : GANDOLFI et al., 1984 ; CICCOTTI et al., 1995). Pour ce qui est de la côte méditerranéenne française, la pêche à la civelle y étant interdite, aucune donnée de pêcherie commerciale n'est disponible. A l'heure actuelle, 
seules deux séries de travaux se sont intéressées au recrutement des civelles, toutes deux en région Languedoc-Roussillon (GANDOLFI HORNYOLD, 1933, 1936 ; puis LECOMTE-FINIGER, 1976, 1983).

La présente étude a été menée dans le delta du Rhône (île de Camargue), et se veut de répondre à diverses interrogations. Dans un contexte de déclin généralisé des effectifs en civelles, il est impératif d'optimiser le recrutement en ouvrant les voies migratoires. Pour cela, il faut acquérir des données fiables et chiffrées sur la temporalité du recrutement. Ces données devraient permettre de proposer des mesures de gestion appropriées quant à l'ouverture des martelières qui régulent les échanges d'eau entre la mer et les lagunes intérieures. D'un point de vue biologique, il est intéressant de caractériser les civelles en recrutement (en terme de biométrie et de pigmentation), et ainsi de voir si les données de Méditerranée française se conforment à celles obtenues en estuaire sur la façade atlantique. L'idée sous-jacente étant que les choses peuvent être très différentes si l'on considère la Méditerranée dans son ensemble comme un immense estuaire. Dans un troisième temps, le jeu de données sur la biométrie et l'état pigmentaire des civelles sera comparé à celui enregistré il y a plus de 25 ans par LECOMTE-FINIGER (1976).

\section{MATÉRIEL ET MÉTHODES}

\section{Site d'étude}

L'échantillonnage s'est déroulé au grau de la Fourcade sur la commune des Saintes-Maries-de-la-Mer (île de Camargue, Bouches-du-Rhône). C'est un chenal qui met en communication la mer Méditerranée et un complexe lagunaire de 11000 ha (étangs des Impériaux, du Vaccarès). Ce chenal constitue la seule voie de passage pour toutes les espèces de poissons et d'invertébrés migrant entre le milieu marin et les lagunes intérieures. Les flux d'eau sont régulés par un système de 13 martelières dont la gestion est confiée au Syndicat de la Digue à la Mer (agriculteurs-riziculteurs des communes environnantes).

Les civelles sont collectées au niveau d'un canal reliant le chenal (et donc la mer) et un système d'évacuation d'eau en provenance des fossés de la commune des Saintes-Maries-de-la-Mer. Lorsque la pompe d'évacuation est en marche (fonctionnement journalier discontinu), il se crée un courant d'eau sortant qui fonctionne comme un débit d'attrait pour les civelles se trouvant en mer ou dans le chenal. Un piège à fin maillage $(1 \mathrm{~mm})$, spécialement conçu aux dimensions du canal $(115 \mathrm{x}$ $90 \mathrm{~cm}$ ), est posé à cet endroit.

\section{Protocole}

Des échantillonnages hebdomadaires ont été effectués de début novembre 2000 à fin mai 2001. La température et la conductivité ont été relevées avant chaque séance de pêche. Les heures de pêche ont évolué avec la photopériode de manière à systématiquement commencer $1 \mathrm{~h}$ avant le coucher du soleil. Le piège est relevé toutes les $30 \mathrm{mn}$ et ce pendant $4 \mathrm{~h}$. Le nombre de civelles est compté après chaque relevé de piège. Un effectif d'environ 20 civelles est formolé sur place $(10 \%)$ et sera ramené au laboratoire pour examen biométrique et pigmentaire. L'excédent est immédiatement relâché en amont, dans l'étang des Impériaux. Lorsque les effectifs de capture sont nuls ou que les conditions de courant ne permettent pas la pose du piège, le souséchantillonnage de 20 civelles est assuré par pêche à l'épuisette.

Les données morphométriques et pigmentaires sont recueillies le lendemain de la pêche, conformément à la méthode de travail de LECOMTE-FINIGER (1976). Le temps 
de conservation des civelles dans le formol est inférieur à $24 \mathrm{~h}$ dans les deux études. Les civelles sont mesurées (longueur totale en $\mathrm{mm}$ ), pesées (masse essuyée en g) et le stade de pigmentation est déterminé selon la classification en usage de ELIE et al. (1982). A noter que ces auteurs ont restreint les limites du stade VI $A_{1}$, et introduit un nouveau stade (VI $A_{0}$ ) par rapport aux classifications précédentes, et notamment par rapport à celle de STRUBBERG (1913) qui a servi de référence à LECOMTE-FINIGER (1976).

\section{Analyse des données}

Les effectifs des captures sont analysés par mois, avec comme unité d'effort le nombre de relevés du piège (normalement huit par échantillonnage hebdomadaire). Les données de longueur et de masse sont comparées entre tous les mois par le test non paramétrique de Kruskal-Wallis (la condition d'homogénéité des variances entre les groupes à comparer n'étant pas vérifiée : test de Levene, $P<0.05$, voir SCHERRER, 1984). L'évolution de ces valeurs biométriques au cours des mois d'échantillonnage est testée par le test de rang de Spearman $(R)$.

Les deux jeux de données à 25 ans d'intervalle (1974-75 vs 2000-01) sont testés mois par mois (novembre à mai) pour des différences quant à la composition pigmentaire et aux valeurs de longueur et de masse (les effectifs de captures ne pouvant être comparés en raison de méthodes et d'efforts de pêche différents). Les proportions mensuelles de chacun des stades pigmentaires sont comparées par le test du $\chi^{2}$. Lorsque la comparaison intéresse de faibles effectifs $(N<10)$, le test de $\chi^{2}$ après correction de Yates $\left(\chi_{c}^{2}\right)$ sera utilisé (SCHERRER, 1984). Pour les raisons invoquées précédemment (échelles de classification), les valeurs biométriques entre les deux jeux de données ne peuvent être comparées que pour les stades $V A, V B$ et $V I A_{2}, V I A_{3}$, VI $A_{4}$. Cependant, dans le souci d'éliminer la variabilité liée au temps de résidence des civelles dans le chenal ou près des côtes, seuls les stades dits jeunes ( $V A, V B$ ) seront retenus (LECOMTE-FINIGER et RAZOULS, 1981). La comparaison est basée sur le test $U$ non paramétrique de Mann-Whitney.

\section{RÉSULTATS}

Un total de 8487 civelles a été capturé par le piège au cours de 25 séances de pêche de novembre à mai. Les mesures biométriques et pigmentaires ont été effectuées sur 21 lots de 20 civelles dont 3 lots ont été capturés directement à l'épuisette. Les relevés physico-chimiques, effectués avant chaque séance, fournissent les valeurs suivantes (moyenne [IC $95 \%]$ ) : conductivité $=22,99 \mathrm{mS} / \mathrm{cm}[16,01-29,97]$; température $=15,04^{\circ} \mathrm{C}[12,85-17,24]$.

\section{Temporalité du recrutement}

Des civelles ont été capturées durant toute la période d'échantillonnage de novembre à mai. La grande variabilité observée autour des CPUE moyennes (et notamment en avril à cause de 897 civelles capturées en une seule unité d'effort) incite à raisonner sur les CPUE médianes (Figure 1). Les CPUE maximales ont lieu au milieu de l'hiver avec un pic en janvier-février. A noter qu'un deuxième pic, bien que moins significatif, se distingue en avril. 


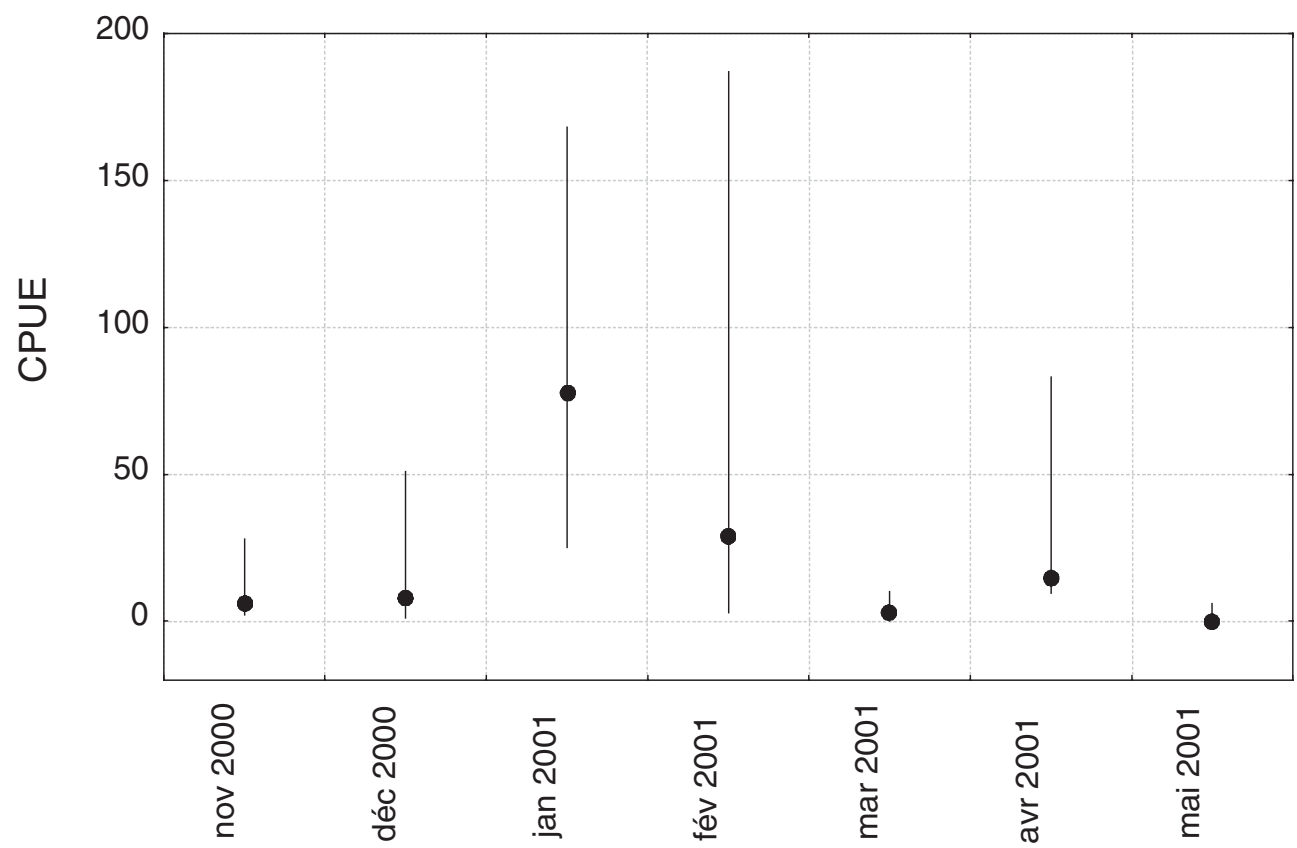

Figure 1

Captures mensuelles par unité d'effort de pêche (médianes $\pm 25 \%$ ).

\section{Figure 1}

Means catches per unit effort of fishing (medians $\pm 25 \%$ ).

\section{Caractéristiques biométriques et pigmentaires}

La longueur des civelles varie au cours de l'année. Des différences significatives de longueur sont observées entre tous les mois (test Kruskal-Wallis : $H(6,426)=$ $144,10, P<0,001)$. Un test de rangs de Spearman révèle une diminution significative des longueurs moyennes au cours des sept mois d'échantillonnage $(R=-0,89, N=7$, $P<0,01)$. La représentation graphique est donnée en Figure $2 \mathrm{~A}$.

Les variations de masse suivent celles observées pour la longueur (Figure 2B). II existe de même des différences significatives entre les valeurs moyennes mensuelles (test Kruskal-Wallis : $\mathrm{H}(6,426)=225,79, P<0,001)$, et une corrélation significativement négative avec les mois $(R=-0,93, N=7, P<0,01)$.

Concernant les stades pigmentaires (Figure 3), on note tout d'abord la très faible présence de civelles au stade $\mathrm{V} A$ tout au long de la période d'échantillonnage (maximum $8 \%, N=6$ en novembre). Les stades pigmentaires les plus avancés, à savoir VI A3 et VI A4, sont eux aussi très peu représentés (maxima respectifs $4 \%$ et $2 \%$ ) et ne sont observés qu'en fin de période d'échantillonnage (respectivement à partir de février et d'avril). Le stade $\mathrm{V} B$ est observé chaque mois et prédomine largement (56\% de l'échantillonnage total, avec des valeurs mensuelles variant de $90 \%$ en décembre à $20 \%$ en mars). En fait à partir du mois d'avril, il semble possible de caractériser l'arrivée d'un second flux de civelles. Cela se traduit par une reaugmentation de la proportion des stades dits jeunes ( $\mathrm{V} B, \mathrm{VI} \mathrm{A}_{0}$ ), et une diminution concomitante des stades plus avancés $\mathrm{VI} \mathrm{A}_{1}$ et $\mathrm{VI} \mathrm{A}_{2}$. 

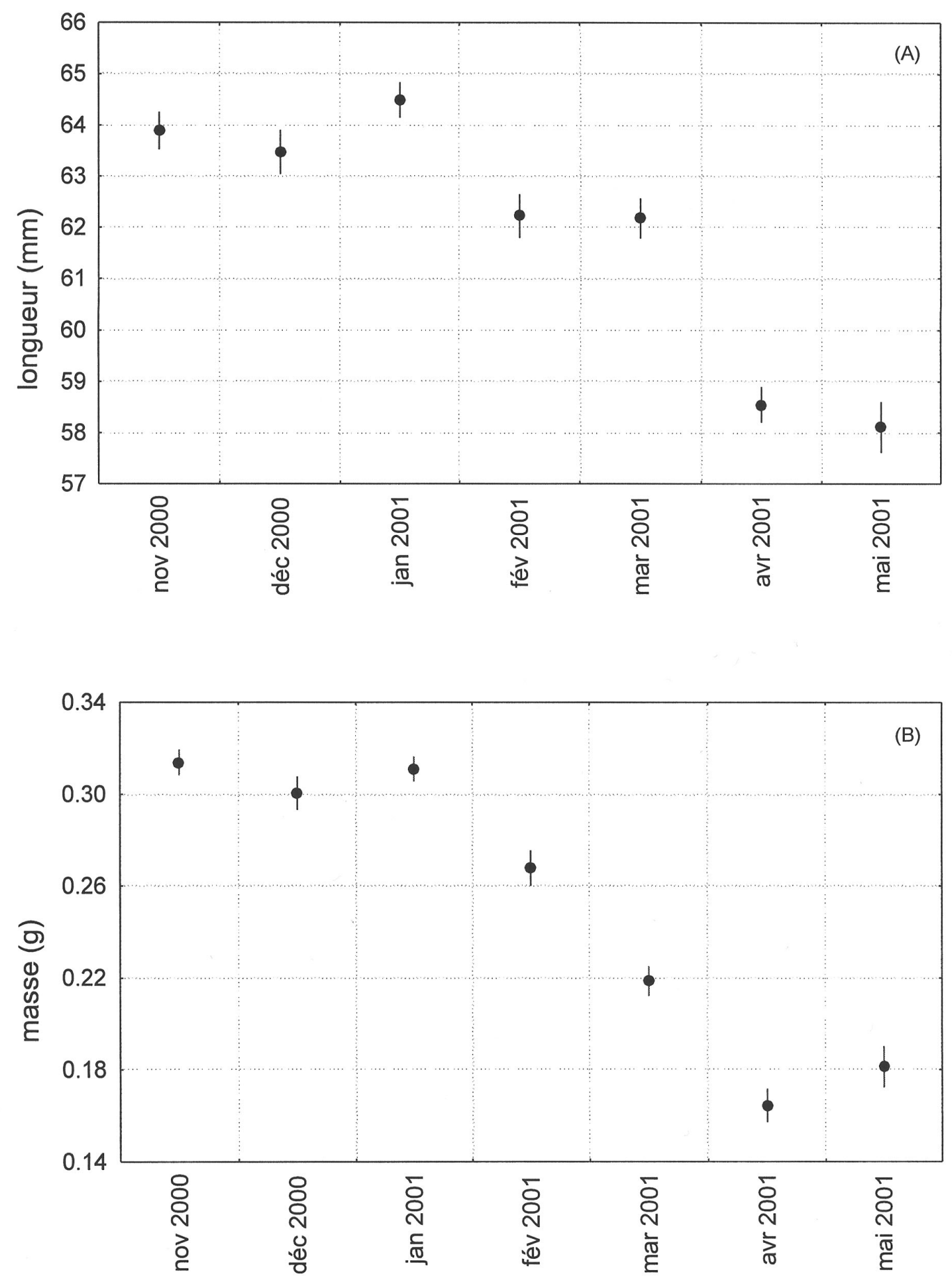

\section{Figure 2}

Évolution des longueurs moyennes mensuelles ( \pm erreur type) (A), et des masses moyennes mensuelles ( \pm erreur type) (B), au cours de la période d'échantillonnage.

\section{Figure 2}

Change in the monthly mean lengths ( \pm standard error) $(A)$, and monthly means weights ( \pm standard error) $(B)$, throughout the sampling period. 


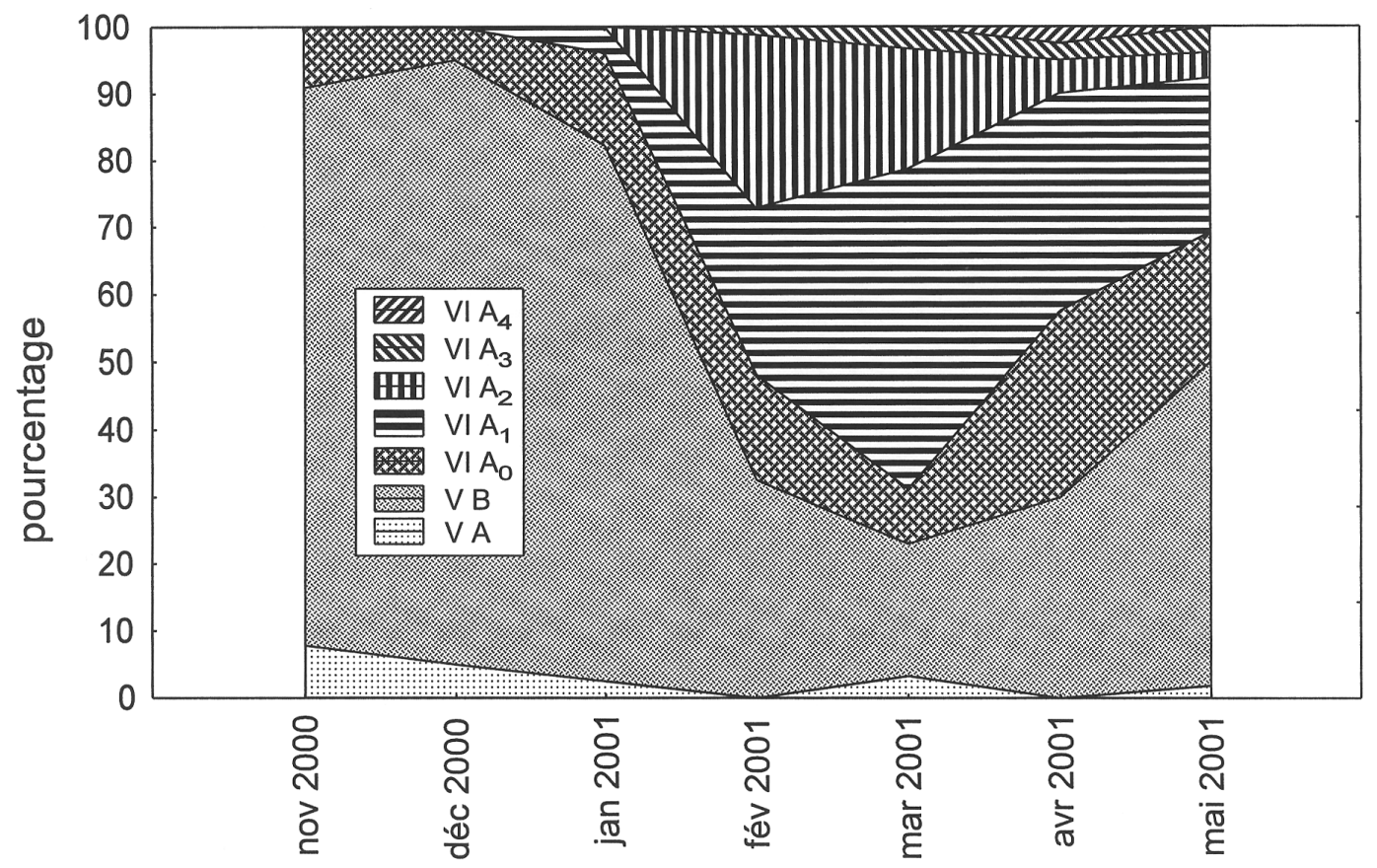

Figure 3

Evolution des stades de pigmentation au cours de la période d'échantillonnage

\section{Figure 3}

Change in the stages of pigmentation throughout the sampling period.

\section{Analyse comparée : saison $1974-75$ vs saison $2000-01$}

Les proportions mensuelles de civelles au stade V B pour la saison 1974-75 et la saison 2000-01 sont consignées dans le Tableau I. II existe des différences significatives pour chacun des mois considérés, à l'exception du mois de mai. La dynamique d'arrivée des civelles est donc totalement différente entre les deux saisons d'échantillonnage.

Les données biométriques de longueur et de masse sont présentées mois par mois pour le stade $\mathrm{V} B$ (Figure 4). L'analyse comparée des données mensuelles montre une très nette tendance à une diminution de la longueur des civelles. Lorsque la comparaison est faite, non plus mois par mois, mais sur la saison (saison 1974-75 vs 2000-01), les données sont les suivantes : $65,4 \pm 5,1 \mathrm{~mm}$ vs $62,9 \pm 3,7 \mathrm{~mm}$; MannWhithney : $U=36108, P<0,001$. Cela correspond à une diminution de longueur de $5,1 \%$ au cours des 25 dernières années.

Pour ce qui est des masses, les analyses comparées mensuelles montrent des résultats variables. Pour les mois de décembre et mai, les civelles de la saison 1974-75 sont significativement plus grosses, mais la tendance est inversée pour les mois de février et mars. En fait, sur l'ensemble des mois considérés, les civelles au stade V B de la période 2000-01 sont significativement plus grosses : $0,26 \pm 0,09 \mathrm{~g}$ vs $0 ., 29 \pm 0,07 \mathrm{~g}$, $U=27404, P<0,001$. 


\section{Tableau 1}

Proportions de civelles au stade V B dans les échantillonnages mensuels. Les comparaisons sont effectuées par un test de $\chi^{2}\left(\chi_{\mathrm{c}}^{2}\right.$ : valeur du Chi-carré après correction de Yates pour les petits échantillonnages).

\section{Table 1}

Proportions of glass eels at the V B stage in the monthly samplings. Comparisons are performed by $\chi^{2}$ test $\left(\chi_{c}^{2}\right.$ : value of Chi-square after Yates correction for small samples size).

\begin{tabular}{llll} 
& $\%$ \% B 1974-75 & $\% \vee B$ B 2000-01 & tests de comparaison \\
\hline novembre & $1 / 6: 16,7$ & $64 / 77: 83,1$ & $\chi^{2}=10,82, P<0,001$ \\
décembre & $110 / 221: 49,8$ & $36 / 40: 90,0$ & $\chi^{2}=22,24, P<0,001$ \\
janvier & $83 / 212: 39,2$ & $52 / 79: 79,7$ & $\chi^{2}=37,94, P<0,01$ \\
février & $56 / 104: 53,8$ & $25 / 77: 32,5$ & $\chi^{2}=10,13, P<0,01$ \\
mars & $37 / 101: 36,6$ & $12 / 61: 19,7$ & $\chi^{2}=5,19, P<0,05$ \\
avril & $88 / 101: 87,1$ & $12 / 40: 30,0$ & $\chi^{2}=5,76, P<0,05$ \\
mai & $54 / 91: 49,8$ & $25 / 52: 48,1$ & $\chi^{2}=0,50, P<0,48$ \\
\hline
\end{tabular}

\section{DISCUSSION}

Le pic d'abondance en civelles au niveau du grau de la Fourcade (commune des Saintes-Maries-de-la-Mer) est observé pour les mois de janvier-février. Ce résultat est en accord avec les données locales (pêcheurs-braconniers, com. pers. ; voir aussi CRIVELLI, 1981) qui situent l'arrivée des civelles au milieu de I'hiver. Le recrutement peut cependant s'opérer sur une période beaucoup plus longue, puisque des civelles ont été capturées sur toute la période d'échantillonnage (novembre à mai).

En fait, au niveau de la mer des Sargasses, des larves leptocéphales d'environ $5 \mathrm{~mm}$ sont observables toute l'année, ce qui suggère une période de reproduction très étalée dans le temps (BOETIUS et HARDING, 1985). Les estimations basées sur le rétro-calcul en otolithométrie s'accordent toutefois avec une intensité reproductive maximale au printemps (LECOMTE-FINIGER et al., 1993 ; Mc CLEAVE, 1993). Considérant une durée de migration trans-océanique de l'ordre de neuf mois (LECOMTE-FINIGER, 1992 ; GUERAULT et al., 1992), cela expliquerait les pics d'abondance observés sur nos côtes en hiver. D'autre part, selon BOETIUS et BOETIUS (1989), la migration trans-océanique des civelles se ferait par vagues successives, hypothèse que semblent confirmer plusieurs études sur la dynamique de recrutement, tant sur la façade méditerranéenne (CICCOTTI et al., 1995) que sur la façade atlantique (CANTRELLE, 1984). Nos données vont également dans ce sens. Un deuxième pic d'abondance, bien que moins significatif, a également été enregistré en avril. Par ailleurs, l'analyse des stades pigmentaires confirme qu'une recrudescence en civelles jeunes (stade V B et VI $A_{0}$ ) a effectivement lieu aux environs du mois d'avril.

Avant l'arrivée de ce deuxième flux migratoire, sur la période de novembre à mars, la proportion de civelles aux stades $\mathrm{V} B$ et $\mathrm{VI} \mathrm{A}_{0}$ va décroissante. En utilisant l'avancement de l'état de pigmentation comme un indice du temps écoulé depuis la métamorphose du leptocéphale en civelle (talus continental), on peut conclure à un vieillissement du stock pendant cette période. L'hypothèse la plus parcimonieuse et la plus couramment avancée pour expliquer l'évolution saisonnière de la pigmentation serait l'existence d'une vie marine post-métamorphique plus longue pour les derniers arrivants. Les raisons pourraient être d'ordre courantologique (MARTIN, 1995 ; JELLYMAN et al., 1999). 

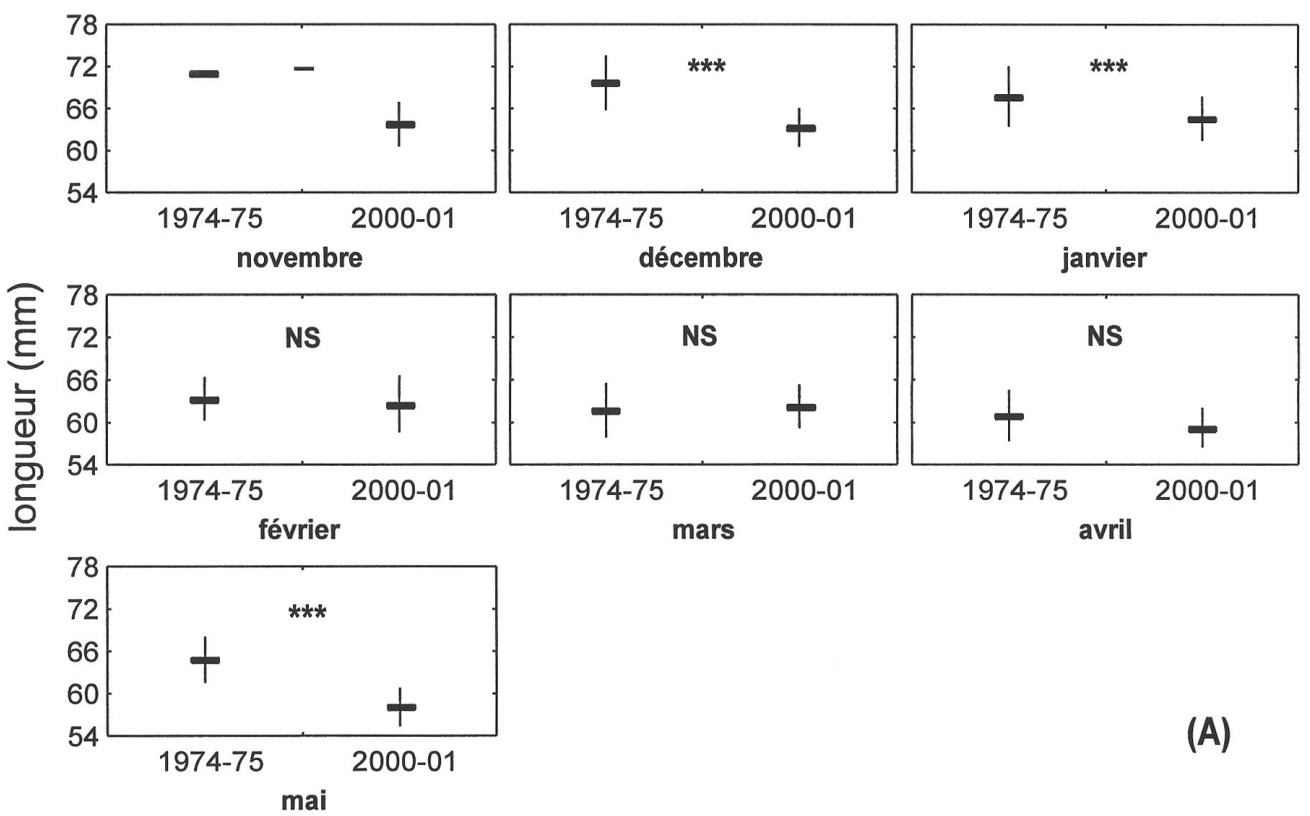

(A)
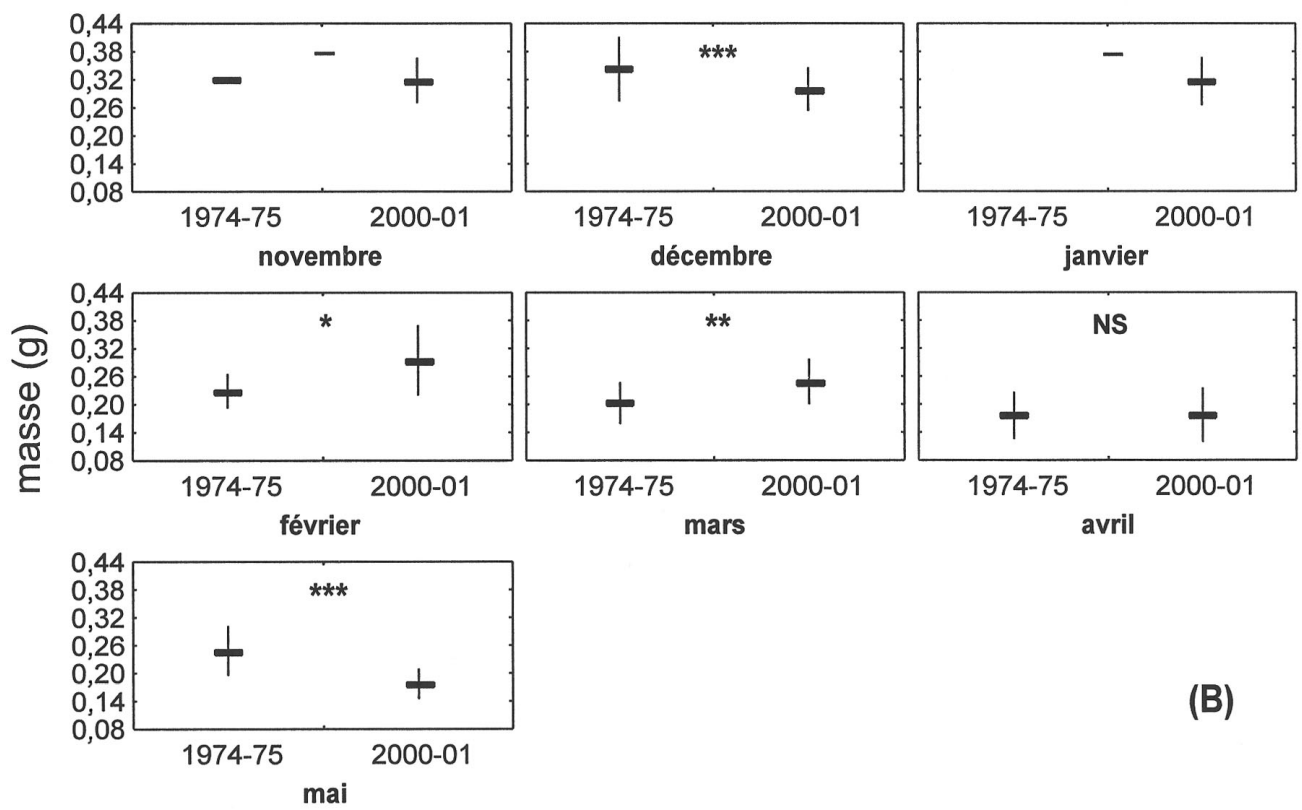

(B)

\section{Figure 4}

Présentation et comparaison statistique des données biométriques mensuelles des civelles au stade V B (saison 1974-75 vs saison 2000-01). (A) longueur moyenne \pm écart type, $(B)$ masse moyenne \pm écart type. ${ }^{\star \star \star}: P<0,001,{ }^{\star \star}: P<0,01,{ }^{*}$ : $P<0,05$, ns : non significatif, - : test non applicable.

\section{Figure 4}

Presentation and statistical comparisons of the monthly biometrics data for glass eels et V B stage (seasons 1974-75 vs season 2000-01). (A) mean length \pm standard deviation, $(B)$ mean weight \pm standard deviation. ${ }^{* * *}: P<0,001,{ }^{* *}$ : $P<0,01,{ }^{*}: P<0,05$, ns : non significant, - : unapplied test. 
Concernant les données biométriques, on observe une diminution saisonnière de la longueur et de la masse des civelles arrivant au grau de la Fourcade. Une telle évolution saisonnière a également été notée par LECOMTE-FINIGER (1976) à BagesSigean. Le phénomène semble même généralisable à l'ensemble des côtes européennes (CHARLON et BLANC, 1982 ; LECOMTE-FINIGER, 1983 ; BOETIUS et BOETIUS, 1989 ; DESAUNAY et GUERAULT, 1997 ; DE CASAMAJOR et al., 2001). Cette diminution des valeurs biométriques reste valable même si l'on ne s'intéresse qu'à un stade pigmentaire donné, en l'occurrence ici, le stade $\mathrm{V} B$. Les raisons seraient à rechercher cette fois dans les ressources planctoniques disponibles au niveau de l'océan et dans des taux de croissance différentiels chez les larves leptocéphales (DESAUNAY et GUERAULT, 1997).

La comparaison des données biométriques, entre la saison 1974-75 (LECOMTEFINIGER, 1976) et la saison 2000-01, révèle une diminution significative des longueurs au stade V B. Les données obtenues sur les masses sont assez curieuses (diminution, augmentation puis diminution), mais considérant les fluctuations possibles dans la prise de cette mesure (évaporation d'eau avant ou pendant la pesée), les résultats ne seront pas discutés plus longuement. La diminution de longueur est évaluée à $5 \%$ sur un intervalle de temps de 25 ans (de $65,4 \pm 5,1 \mathrm{~mm}$ à $62,9 \pm 3,7 \mathrm{~mm}$ ). Nous confirmons ainsi sur la façade méditerranéenne, la tendance observée sur la façade atlantique française. Sur l'Adour par exemple (sud-ouest de la France), les longueurs et masses moyennes de civelles au stade V B sont passées de $76 \mathrm{~mm}$ et $0.39 \mathrm{~g}$ pour la saison 1979-1980 à $67 \mathrm{~mm}$ et $0,30 \mathrm{~g}$ pour la saison 1991-1992, soit des diminutions respectives de 9 et $30 \%$ en 12 ans (DESAUNAY et GUERAULT, 1997). La diminution des valeurs biométriques est aussi signalée sur de nombreux autres estuaires de la façade atlantique et dans un autre site de la façade méditerranéenne (Oued Sebou, Maroc ; YAHYAOUI, 1991). Les conséquences sur la croissance et la survie future des civelles ne sont pas connues, et les causes sont encore très hypothétiques (évolution des courants marins, diminution des ressources trophiques hauturières durant la croissance du leptocéphale, etc...).

\section{CONCLUSION}

Les résultats obtenus sur ce site d'étude de la côte méditerranéenne française vont dans le sens des observations signalées sur les côtes atlantiques, à savoir : i) diminution des masses et des longueurs moyennes des civelles au cours de la saison, ii) diminution des masses et des longueurs, pour un stade donné ( $\mathrm{V} B$ ), au cours de la saison, iii) vieillissement global du stock civellier au cours de la saison, iv) diminution de la taille des civelles au cours des dernières décennies.

Les arrivées massives de civelles, sous réserve d'importantes fluctuations interannuelles, se situant en janvier-février, nous incitons les gestionnaires du grau de la Fourcade (agriculteurs-riziculteurs) à prendre en compte les intérêts des pêcheurs professionnels et à ouvrir un maximum de martelières durant ces deux mois d'hiver.

\section{REMERCIEMENTS}

Nous associons à ce manuscrit toutes les personnes qui ont participé aux sorties nocturnes de la campagne de pêche : ARNAUD DORGERE, CATHERINE VAUFREY, DAMIEN COHEZ, NICOLAS LE CLAINCHE, ANNA SALTMARSH, ERIC LIBERCIER, BLANCA PEREZ, DENIS FATIN, MATHIEU BOSSAERT, JEROME JOURDE, NASSIMA AGHANIM, MICKAEL VEILLE, SOPHIE DE LA BARRE et PASCAL CONTOURNET. Cette étude s'est déroulée dans le cadre d'un stage BTS «Gestion et Protection de la Nature " de l'un d'entre nous (E. SERGENT), dont le support financier a été assuré par la fondation Sansouire. 


\section{BIBLIOGRAPHIE}

BOETIUS I., BOETIUS J., 1989. Ascending elvers, Anguilla anguilla, from five European localities. Analyses of pigmentation stages, condition, chemical composition and energy reserves. Dana, 7, 1-12.

BOETIUS J., HARDING E.F., 1985. A re-examination of Johannes Schmidt's Atlantic eel investigations. Dana, 4, 129-162.

CANTRELLE I., 1984. Les populations de civelles d'Anguilla anguilla L. en migration dans l'estuaire de la Gironde. Vie Milieu, 34, 109-116.

CHARLON N., BLANC J.-M., 1982. Etudes des civelles d'Anguilla anguilla L. dans la région du bassin de l'Adour. 1. Caractéristiques biométriques de longueur et de poids en fonction de la pigmentation. Arch. Hydrobiol., 93, 238-255.

CICCOTTI E., RICCI T., SCARDI M., FRESI E., CATAUDELLA S., 1995. Intraseasonal characterization of glass eel migration in the river Tiber: space and time dynamics. J. Fish Biol., 47, 248-255.

CRIVELLI A.J., 1981. Les peuplements de poissons de la Camargue. Rev. Ecol., 35, 617671.

CRIVELLI A.J., 1998. L'anguille méditerranéenne : synthèse bibliographique. Rapport du COGEPOMI du bassin Rhône-Méditerranée-Corse, $83 \mathrm{p}$.

DE CASAMAJOR M.N., LECOMTE-FINIGER R., PROUZET P., 2001. Détermination de l'état d'amaigrissement des civelles (Anguilla anguilla) en migration en zones côtière et estuarienne. C.-R. Acad. Sci. Paris, 324, 345-353.

DESAUNAY Y., GUERAULT D., 1997. Seasonal and long-term changes in biometrics of eel larvae: a possible relationship between recruitment variation and north Atlantic ecosystem productivity. J. Fish Biol., 51, 317-339.

DUSSERRE K., LOSTE C., 1997. La pêche sur les étangs de Gruissan. Rapport du Centre d'Etudes et de Promotion des Activités Lagunaires et Maritimes CEPRALMAR, $30 \mathrm{p}$.

ELIE P., LECOMTE-FINIGER R., CANTRELLE I., CHARLON N., 1982. Définition des limites des différents stades pigmentaires durant la phase civelle d'Anguilla anguilla. Vie Milieu, 32, 149-157.

EZZAT A., EL SERAFY S., 1977. The migration of elvers of Anguilla anguilla L. in the Mer canal, Alexandria, Egypt. J. Fish Biol., 11, 249-256.

GANDOLFI HORNYOLD A., 1933. Mensurations de civelles de Sète et de la Loire, pêchées les 18 et 19 mars 1932. Arch. Zool. Exp. Gén., 75, 359-367.

GANDOLFI HORNYOLD A., 1936. La civelle de Sète de décembre 1934 à mars 1935. Bull. Soc. Hist. Nat. Toulouse, 69, 69-76.

GANDOLFI G., PESARO M., TONGIORGI P., 1984. Environmental factors affecting the ascent of elvers, Anguilla anguilla (L.), into the Arno river. Oebalia, 10, 17-35.

GUÉRAULT D., LECOMTE-FINIGER R., DÉSAUNAY Y., BIAGIANTI-RISBOURG S., BEILLOIS P., GRELLIER P., 1992. Glass eel arrivals in the Vilaine estuary (northern Bay of Biscay) in 1990: demographic features and early life history. Ir. Fish. Invest. Series A, 36, 5-14.

HELDT H., HELDT H., 1929. Etudes sur les civelles de Sidi Daoud. Bull. Sth Océanogr. Salammbô, 16, 1-23.

JELLYMAN D.J., CHISNALL B.L., BONNETT M.L., SYKES J.R.E., 1999. Seasonal arrival patterns of juvenile freshwater eels (Anguilla spp.) in New Zealand. New Zealand J. Mar. Freshw. Res., 33, 249-261.

LECOMTE-FINIGER R., 1976. Contribution à l'étude biologique et écologique des civelles (Anguilla anguilla Linné 1758) lors de leur pénétration dans un étang méditerranéen. Vie Milieu, 26, 123-144.

LECOMTE-FINIGER R., 1983. Etude morphométrique et énergétique de la civelle de méditerranée au cours de la pigmentation. Vie Milieu, 33, 87-92.

LECOMTE-FINIGER R., 1992. Growth history and age at recruitment of European glasseels (Anguilla anguilla) as revealed by otolith microstructure. Mar. Biol., 114, 205-210. 
LECOMTE-FINIGER R., BRUSLÉ J., 1984. L'anguille Anguilla anguilla des lagunes du Languedoc-Roussillon : intérêt biologique et halieutique. Vie Milieu, 34, 185-194.

LECOMTE-FINIGER R., DESAUNAY Y., GUERAULT D., GRELLIER P., 1993. The immigration of $A$. anguilla in coastal waters: questions about the determinism of the otolith structures. EIFAC, Working Party on eel, Olsztyn, Poland.

LECOMTE-FINIGER R., RAZOULS C., 1981. Influence des facteurs hydrologiques et météorologiques sur la migration anadrome des civelles dans le golfe du Lion. Cah. Lab. Montereau, 12, 13-16.

MARTIN M.H., 1995. The effects of temperature, river flow, and tidal cycles on the onset of glass eel and elver migration into fresh water in the American eel. J. Fish Biol., 46, 891-902.

Mc CLEAVE J.D., 1993. Physical and behavioural controls on the oceanic distribution and migration of leptocephali. J. Fish Biol., 43, 243-273.

MORIARTY C., DEKKER W., 1997. Management of the European eel. Fish. Bull., 15, 1110.

QUIGNARD J.P., MAZOYER C., VIANET R., MAN WAI R., BENHARRAT K., 1983. Un exemple d'exploitation lagunaire en Languedoc : l'étang de l'Or (Mauguio). Pêche et production halieutique. Science Pêche, Bull. Inst. Pêches marit., 336, 3-23.

SCHERRER B., 1984. Biostatistiques. Gaëtan Morin, Chicoutimi, Canada, 850 p.

STRUBBERG A., 1913. The metamorphosis of elvers as influenced by outward conditions. Some experiments. Meddr Komm. Havunders. Ser. Fisk., 4, 1-11.

YAHYAOUI A., 1988. Etude comparée des civelles d'anguilles (Anguilla anguilla) dans trois sites méridionaux (atlantique et méditerranéens). Inv. Pesq., 52, 501-515.

YAHYAOUI A., 1991. Contribution à l'étude de la biologie de l'anguille (Anguilla anguilla L. 1758) dans son aire méridionale de répartition géographique : littoral atlantique et méditerranéen marocain. Thèse, Université de Rabat, Maroc, $171 \mathrm{p}$. 